\title{
Line integrated traffic operation assistance system
}

\author{
H. Ochiai, T. Nakamura \& M. Kitai \\ Technical Research and Development Department, \\ West Japan Railway Company, Japan
}

\begin{abstract}
The West Japan Railway Company is developing a traffic operation assistance system in cases of abnormal traffic congestion. The main target of this system is, when an abnormal situation arises in traffic operation, to establish a rescheduled diagram as quickly as possible and to rapidly transmit it and the certain execution of it. For this purpose, this system supports three types of assistance functions such as grasping the situation and rescheduling and instruction/notification sending in cases of abnormal traffic congestion. Also, to estimate objectively the effect of these assistance functions, we made a real time train traffic simulator to precisely simulate an abnormal traffic situation. The assessment result, applying this simulator, showed a dramatic reduction of the operator's work processes and the factors of communication loss and mistreatment may also be reduced.

Keywords: programmed route control system, rescheduling, simulator.
\end{abstract}

\section{Introduction}

The West Japan Railway Company is developing the Next Generation Integrated Traffic Operation Management System. The central function of this system is the assistance of a traffic operator in cases of abnormal traffic congestion.

The most crowded and mass traffic volume area on West Japan Railway lines is the urban area located with Osaka as the centre. In this area, many trains run through complexly and mutually over several lines (see fig. 1). Even in this traffic operation under normal conditions according to the planned traffic diagram, the operators are just observing the traffic situation because the traffic is automatically controlled by the programmed traffic control system. But once abnormal conditions like an accident or weather disaster occur, immediately the effect of 
these situations spread everywhere in the urban area, the required traffic operation suddenly increases such as the many communications, instructions, notifications and affirmations that will induce rapid operation and mistreatment. Also if an additional incident simultaneously overlaps, further confusion will arise. For this reason, we decided to develop a new supporting system for the traffic operation in cases of abnormal congestion.

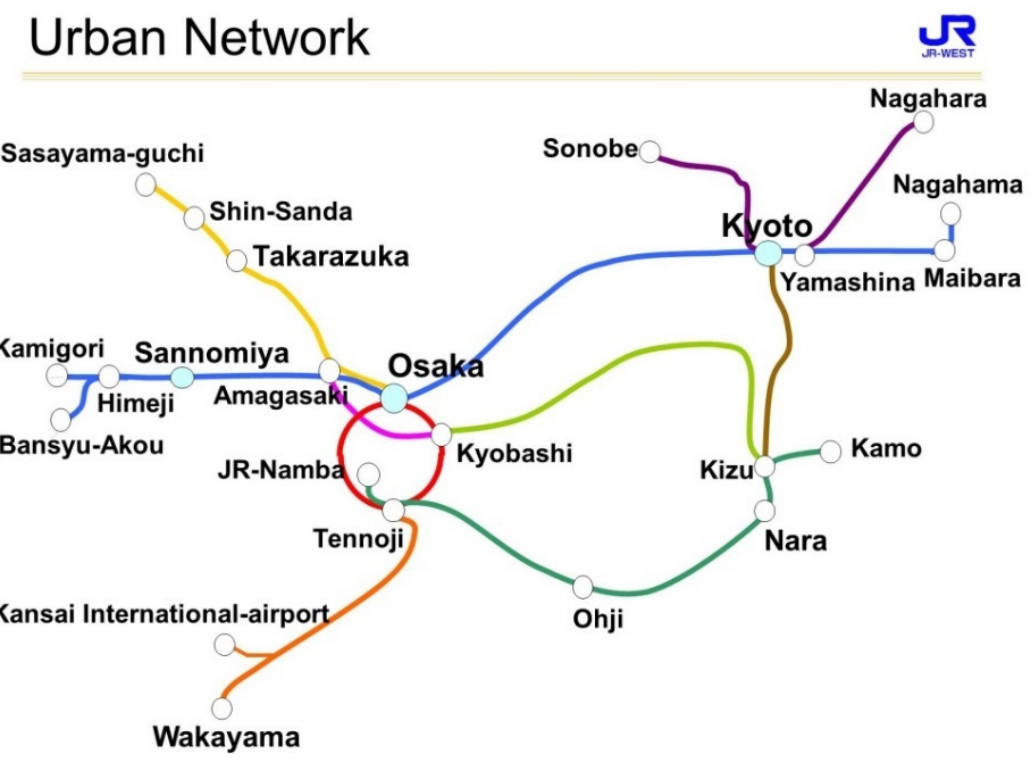

Figure 1: Urban area network.

\section{The new operation method in abnormal situations}

The target result of this system is when great confusion occurs in the traffic, to make a rescheduled diagram as soon as possible, transmitting this revised diagram to the relating sections, executing precisely according to this new diagram. This concept also stands for the passenger's point of view. It means the most desirable information for the passenger is to not delay time from the original timetable but to have the arrival time of the corresponding train match their aims. For this reason, we think it will be better to present and fix a precisely executable rescheduled diagram and to execute it exactly according to the new diagram rather than always changing or alternating diagrams with the aim of a very quick recovery.

\section{Traffic operator's action needed in abnormal traffic congestion}

Analysing the traffic operator's action in abnormal situations is to realize the abovementioned basic concept. We summarize the following three categories of functions. 


\subsection{Situation grasp}

The situation grasp phase provides information to understand the objective condition in abnormal circumstances. When train traffic operation abnormality happens, at the first instance, it needs a correct and speedy grasp of the situation and the operator's appropriate response must be made according to such a situation.

\subsection{Rescheduling diagram}

In cases of traffic diagram congestion, immediate traffic diagram rescheduling by the train returning to the station having returnable facilities outside the obstacle section and train suspension shall be executed and the required rescheduling of the traffic diagram implemented after predictive obstacle release timing.

\subsection{Instruction/notification sending}

Currently, the notification sheet is handed to the driver at the station or communication by train radio is done. As a consequence, the operator and station staff's load increases, resulting in a lack of or delay in sending a notification. This situation can be changed by the automatic finding of the target train and the direct data transmission to the object train's on-board display. It will realize quick and certain instruction/notification sending.

\section{System configuration method}

Current Programmed Route Control systems are configured as all functions dedicated to each line (see fig. 2(a)). This system configuration does not treat appropriately for inter lines relating functions. So we changed this system configuration to separate the line dedicated function only for the route control function and the other assistance functions are concentrated on the common functions to not dedicate each line. We are going to forward this type of basic configuration for the Next Generation Integrated Traffic Management System (see fig. 2(b)). Though we are aiming at the fig. 2(b) system configuration as the NGITMS, it will take many decades to replace all the Programmed Rout Control systems. So we considered one method which can shift smoothly to the future system configuration still keeping the current system configuration. In this method, the new common function part can input data from the current Programmed Rout Control system and set in the common data. This common data can be accessed from every new common function part. As the common function part, it includes situation grasp and rescheduling and instruction/notification sending (see fig. 3). This system configuration can realize the smooth shift to the NGITMS without changing the current basic system configuration even with step-by-step replacement of the Programmed Rout Control system. 


\section{Current system}

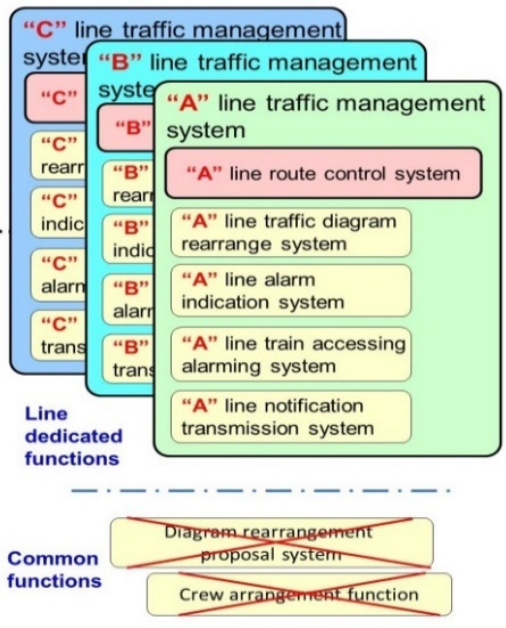

(a)

\section{Next Generation System}

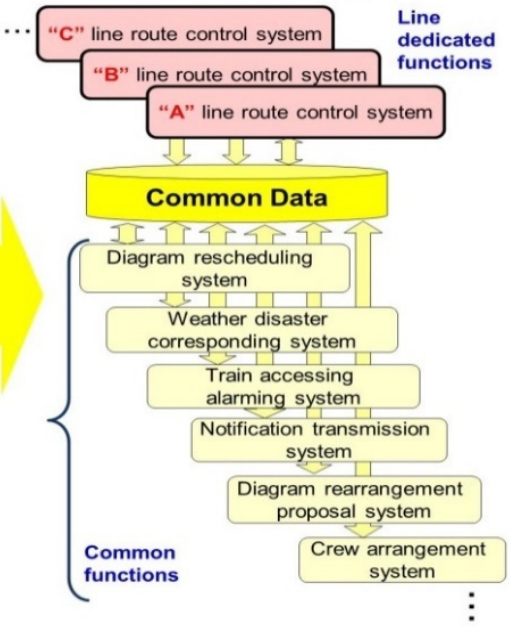

(b)

Figure 2: Current and next generation system.

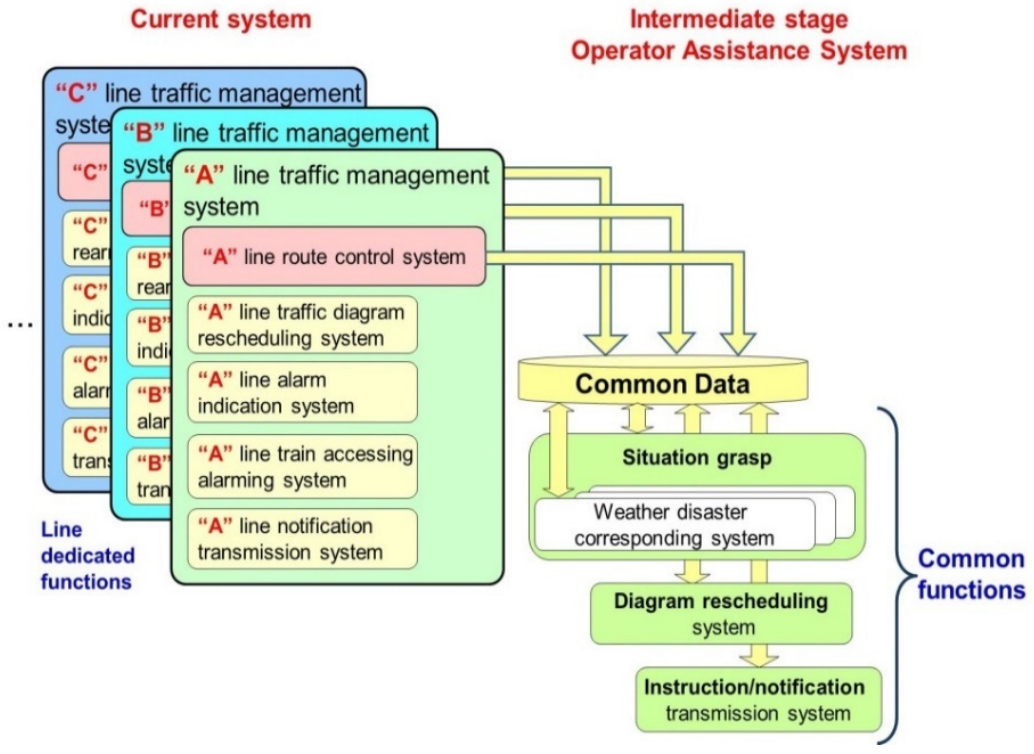

Figure 3: Operator assistance system. 


\section{System configuration}

The system configuration of the new developed lines integrated Traffic Operator Assistance System is shown in fig. 4. In this figure, the Programmed Route Control systems remain as the current system configuration and the new assistance system can input from them and the output way to this machine still remains as the current method. As the assistance system, the situation grasp, the rescheduling. the instruction/notification sending and on-board driver's terminal functions are provided.

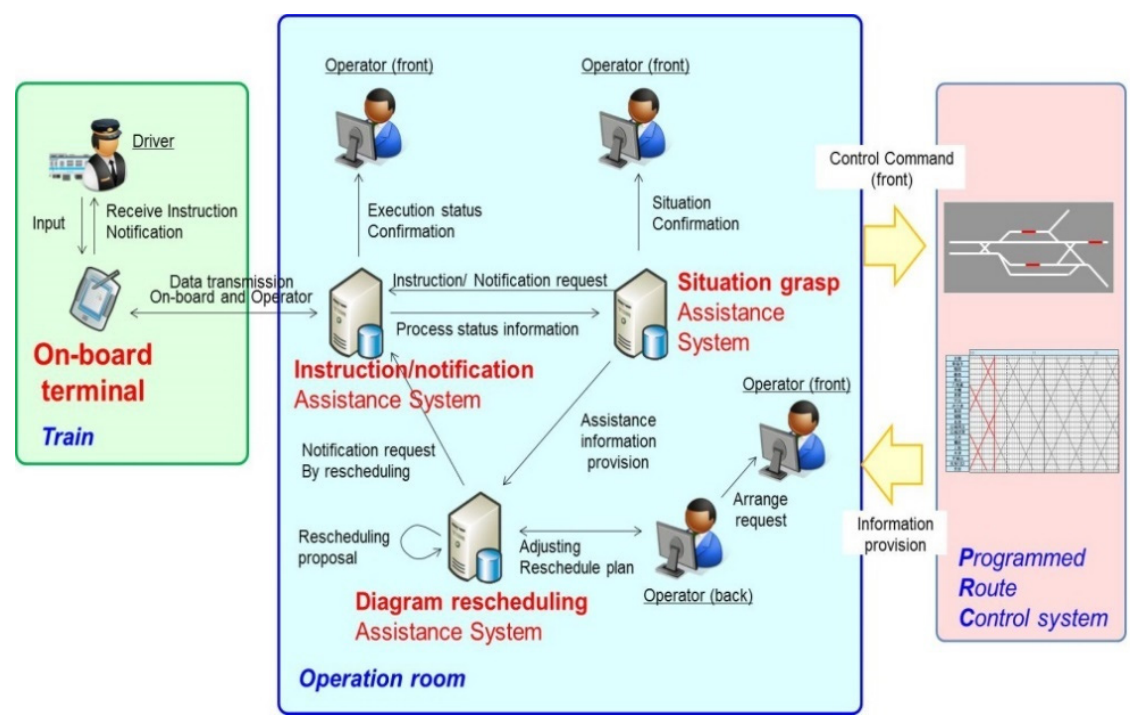

Figure 4: Traffic operator assistance system.

\section{Simulator for system assessment}

We wanted to assess objectively that the new system can be applied efficiently or not in cases of abnormality. For this reason, we also developed a real-time train traffic simulator which precisely reflects abnormal situations for assessment. Of course, an abnormal situation setting is impossible in the real world because of obstacle setting in traffic, so this type of simulator is essential to assess such a situation. The total system configuration is shown in fig. 5. The traffic operation simulator includes many kinds of objects to simulate traffic operation and its action is recorded in real time. By analysing this recorded data, assistance effects will be assessed objectively. There are many simulation objects. Such as the trains, rail condition, signals, stations, programmed traffic control, etc. These objects can be set for any kind of obstacle conditions and simulate the traffic situation precisely in cases of abnormality. For example, trains that run between stations precisely according to the predefined time table in normal conditions (see fig. 6 
upper running curve). But once any traffic regulation is applied to the train in abnormal conditions, the train automatically runs according to such speed restriction (see fig. 6 lower running curve).

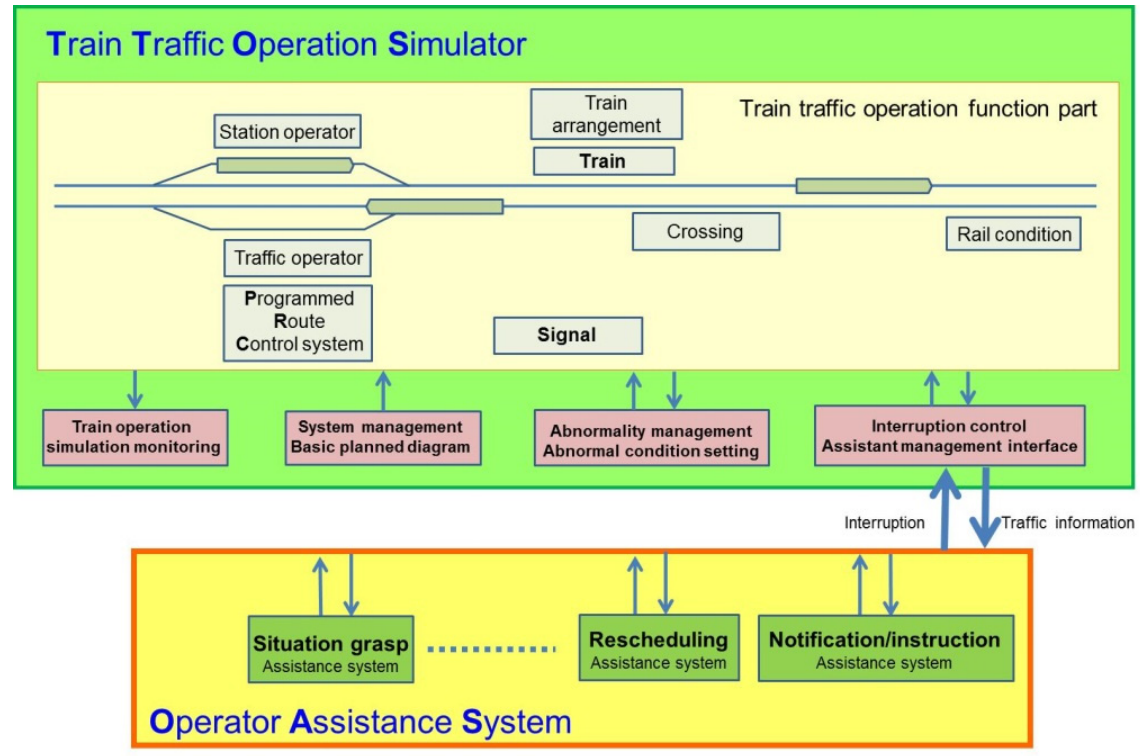

Figure 5: Train Traffic Operation Simulator.

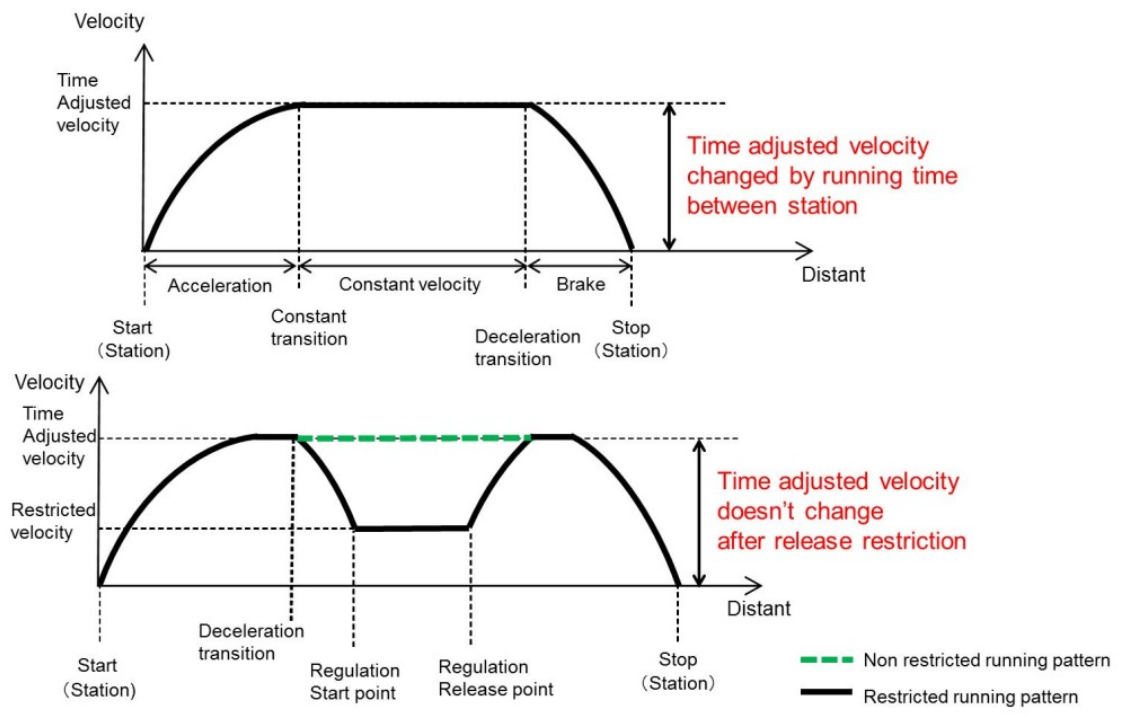

Figure 6: Train running simulation. 


\section{Implementation example}

Implementation examples are shown as follows.

\subsection{Situation grasp}

The situation grasp function can provide the information to grasp a situation in abnormal circumstances objectively. If traffic operation abnormality occurs, a precise grasp of the situation is needed in the first instance and requires the appropriate treatment according to the situation. As the first step implementation, the road crossing obstacle and the body injury accident are selected for its situation setting (see fig. 7). After the operator's entry of the occurred obstacle point, this system will be indicated on the display screen and the position on the rail line alongside, the type of obstacle, affected area, emergency stopping area, head car stopping position, restricted signal status, corresponding required procedure, etc. The operator can correctly recognize the abnormal situation and initiate the next action, such as the object train emergency stop instruction and so on. This result covers inter lines action and efficiently supports the traffic diagram rescheduling and instruction/notification sending. Continuous system support scope will implement more widely like with weather disaster, rain, wind, earthquake, rail temperature, etc. Furthermore, train trouble and catenary power down will also become supporting objects.

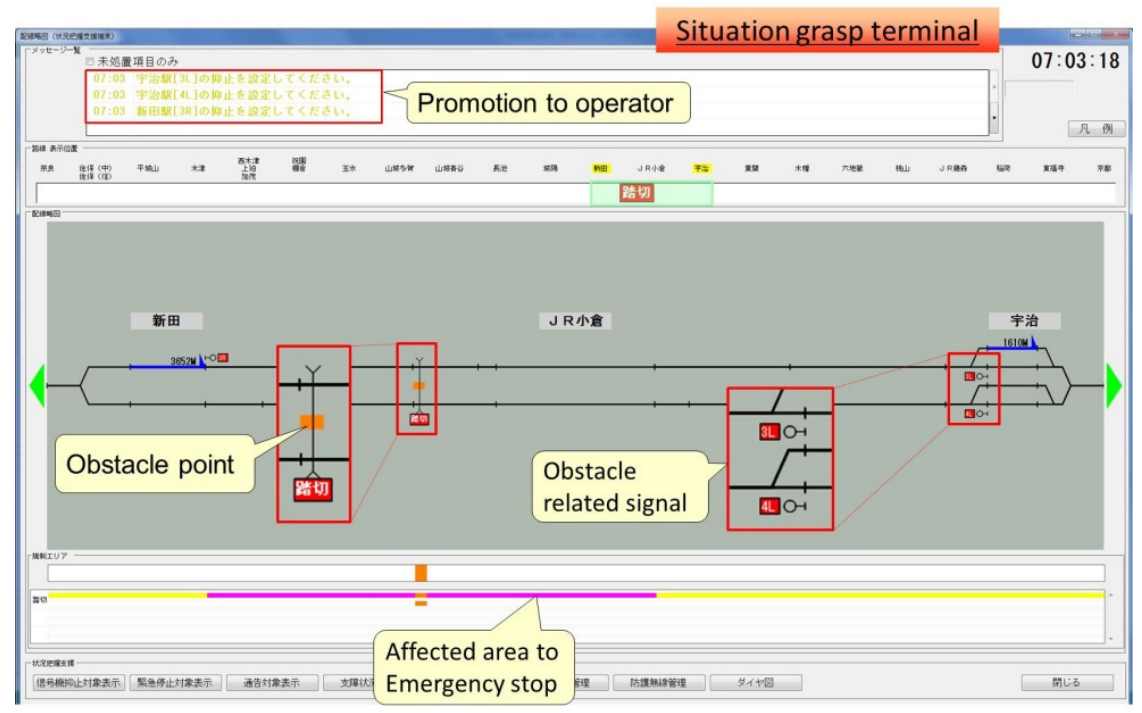

Figure 7: Situation grasp display example. 


\subsection{Traffic diagram rescheduling}

If the traffic diagram is congested by abnormal conditions, the train reverses in order to avoid the obstacle area and train suspension shall be applied as the rescheduling diagram. We have been developing an automatic rescheduling algorithm [1]. For this application, obstacle area and predicted obstacle release timing will be received from the situation grasp function and applying the predicted special reverse and suspend diagram pattern will automatically generate the traffic rescheduled diagram (see fig. 8). A conventionally executed manually treated rescheduling operation will change to this automatic system support. The operator can reschedule according to the proposal from this system output. By this method, currently depending on the many kinds of operator's treatment will change with an operator's minor modification based on the automatic rescheduling proposal (see fig. 9).

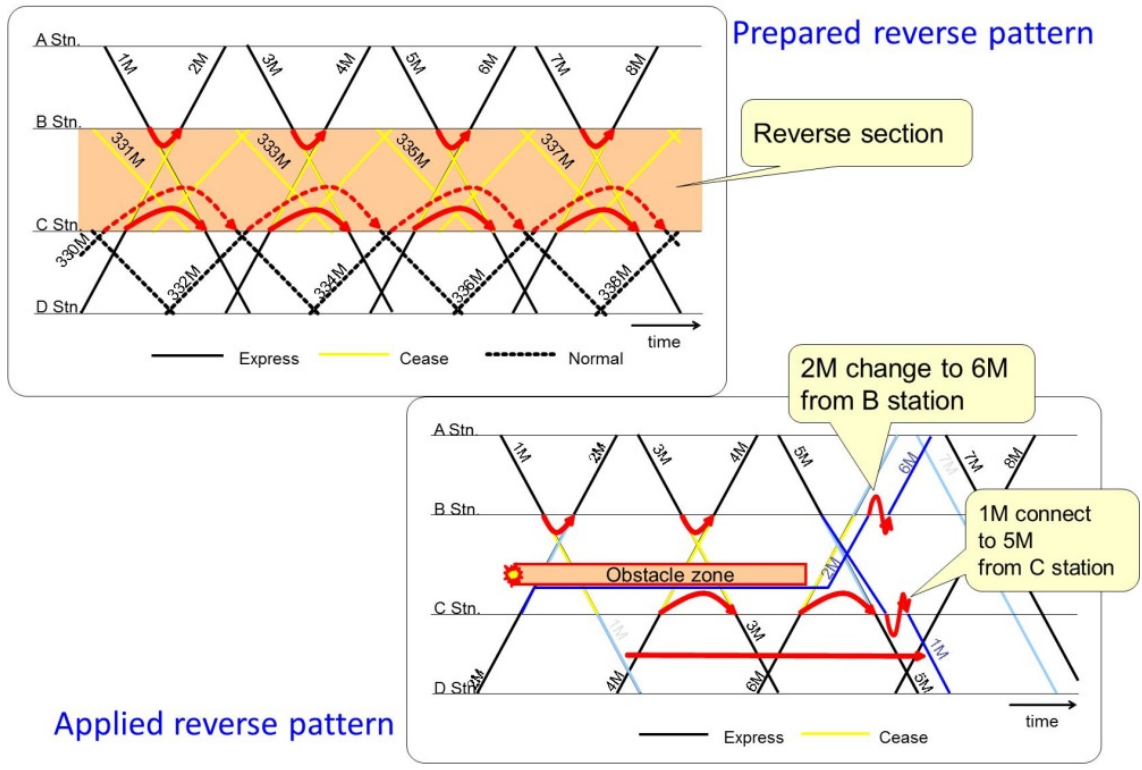

Figure 8: $\quad$ Prepared reverse pattern and applied result.

\subsection{Instruction/notification sending}

Conventionally, notifications are sent by hand from station staff or by train radio communication from the operator. As a result, operator and station staff load are large and mistreatment is induced, such as the lack of notification and delay. These situations will change to automatic notification sent by automatic object train pick up and direct data transmission to the train driver's on-board display. It makes for certainty and speed and no lack in sending (see fig. 10). Furthermore, not only notification sending but also stop instruction and timetable sending which need on-board and ground data transmission will be introduced as the integrated system 


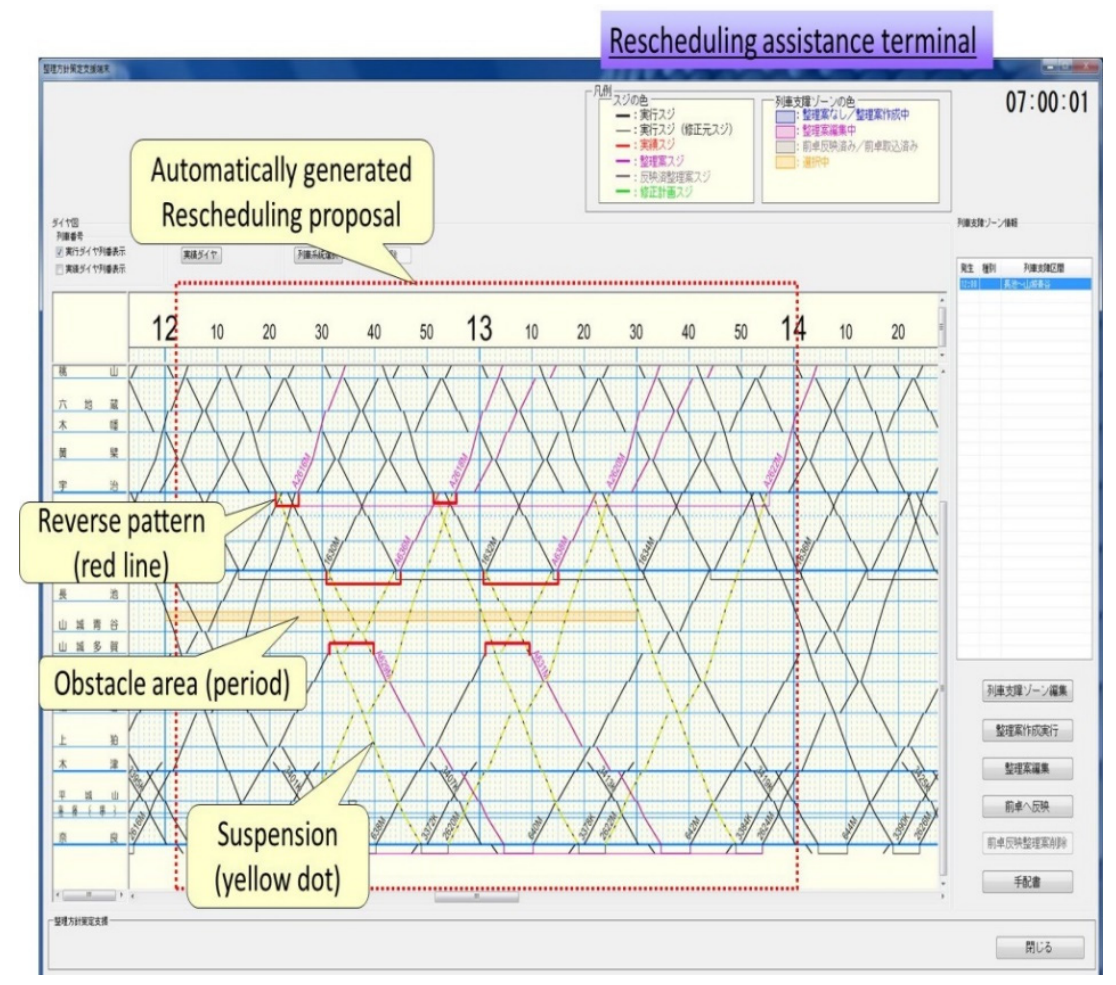

Figure 9: Rescheduling proposal display example.

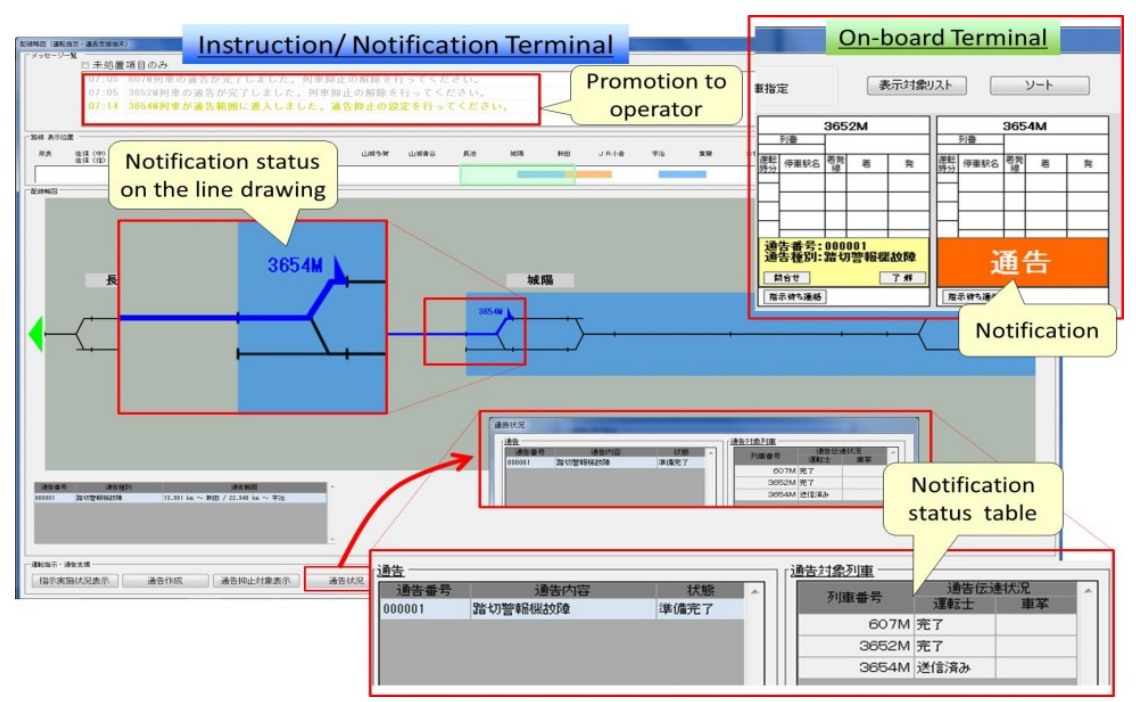

Figure 10: Notification sending display example. 
configuration. The stop instruction applies to the train which requires an emergency stop. The driver receiving this instruction shall immediately stop the train and notify the operator of the train stopping situation. The timetable sending function is the rescheduled timetable transmission to the object train driver. The object train driver can drive the train according to this rescheduled timetable.

\section{Assessment result}

By using the traffic operation simulator, the current operation and the proposed new system's operation are comparatively assessed. The Train Traffic Operation Simulator can provide precise data recording in the simulation process and analyse this data to clarify the operator's load in the case of an abnormal situation. The degree of congestion, bottleneck point, lack of treatment or mistreatment will be assessed objectively. Furthermore, the introduction effects by using a new operator assistance system will clearly compare to the current manual operation process in cases of abnormality. This simulator can pause any timing during the simulation and back to any timing from the captured point every 5 minutes. As a result, the correction of misoperation and other operation treatments can be made again immediately after modifying the situation for efficient simulation. The simulator can create any occasion which is difficult to set in a real operation environment. The abnormal situation can be set in plural and simultaneously. For this reason, the simulator can be used efficiently as an operator exercise in cases needing emergency treatment. This simulator can record the execution process precisely. By analysing this data, objective assessment can be made using this simulator. Fig. 11 shows the operation work process and the time consumption comparison between the current operation and the proposed new system. It shows a dramatic reduction of current operator's work required and the reduced assistance part by the system support. Fig. 12 shows the total work time comparison between the current and the new supporting system.

\section{Impact of innovation}

The result of this system assessment indicates that even if still using the current Programmed Route Control system, the inter line works are efficiently improved by the inclusion of this new system. The introduction of this new system proves system support efficiency instead of the current manually cautioned works by the operator. This result is due to the common functions method covering whole lines. This common function part shall remain utilized in the future, if the Programmed Route Control system will be replaced by the new one, the basic system configuration is still maintained. This new system innovation clearly shows great steps toward the future. 
Current operation

System assisted operation

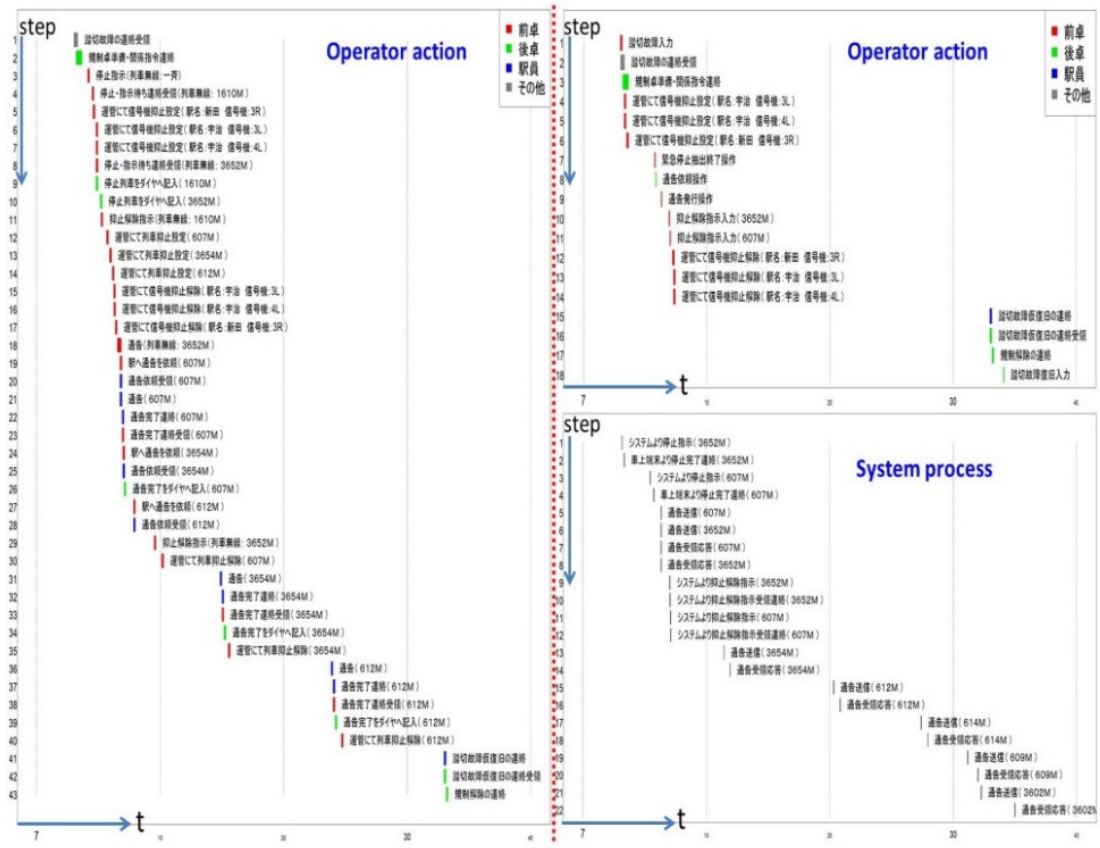

Figure 11: Current operation and system assistance operation.

Accumulated operation time: road crossing failure

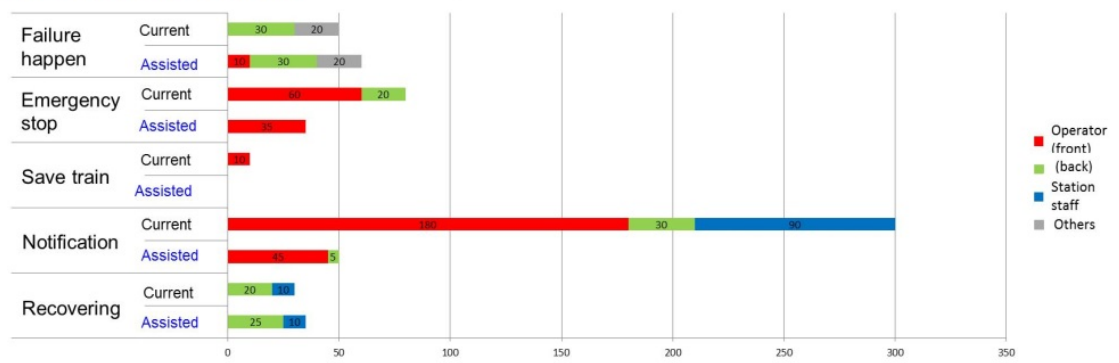

Each actor accumulated operation time: road crossing failure

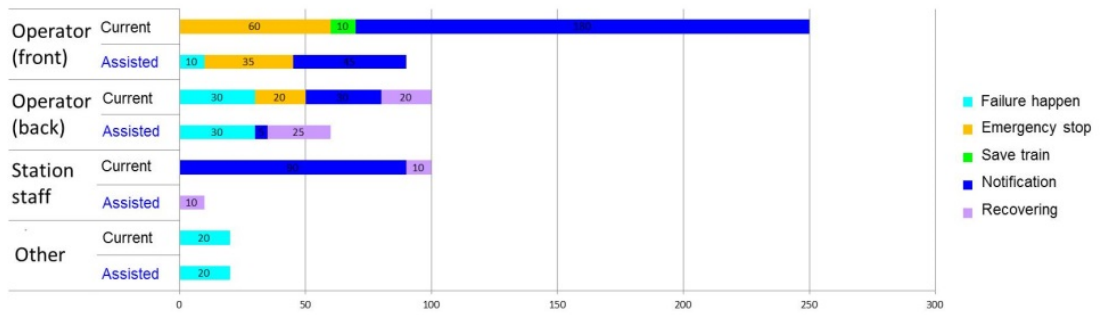

Figure 12: Work time comparison current and assisted. 


\section{Conclusion}

Introduction effectiveness of this system is proved by the result of simulator assessment. This system was made as a prototype in 2015 and is preceding system assessment. In this assessment phase, comparison between the current operation and this new system supported operation will be estimated concretely. After that, the abnormal situation extensions like as weather disaster, earthquake, train trouble, will be added. Furthermore, improvement of Human Machine Interface and reliability will precede and the basic assessment toward the New Generation Integrated Traffic Management System will finish by 2019. After this assessment process, the lines integrated traffic operator assistance system is going to be implemented as part of the New Generation Integrated Traffic Management System.

\section{Reference}

[1] Nakamura T., Hirai C., Nishioka Y., "A Practical Train Rescheduling Algorithm Using Three Predetermined Factors", In proceedings of 4th International Seminar on Railway Operations Modelling and Analysis (Rail Rome 2011), Rome, Italy, February 2011. 